Pacific Journal of Mathematics

ABSOLUTELY DIVERGENT SERIES AND ISOMORPHISM OF 


\title{
ABSOLUTELY DIVERGENT SERIES AND ISOMORPHISM OF SUBSPACES II
}

\author{
William H. Ruckle
}

The following relations between a Banach space $E$ and a Banach space $X$ are, roughly speaking, generalizations of the relation " $E$ is a closed subspace of $X$."

(LIX) The finite dimensional subspaces of $E$ are uniformly isomorphic to subspaces of $X$ under isomorphisms which extend to all of $E$ without increase of norm.

$(\mathrm{SpX})$ Finite rank mappings from any Banach space into $E$ can be uniformly factored through subspaces of $X$.

(ASX) The continuous linear mappings from $E$ into $X$ distinguish the absolutely summing mappings from any Banach space into $E$.

(SIX) For each absolutely divergent series $\Sigma_{n} x_{n}$ in $E$ there is a continuous linear mapping $T$ from $E$ into $X$ such that $\Sigma_{n} T x_{n}$ diverges absolutely.

Our main result is that these four conditions are equivalent if $X$ contains a subspace isomorphic to $\lambda[X]$ where $\lambda$ is a normal $B K$-space. A related result of some interest is that the class of continuous linear mappings which factor through spaces which contain a complemented copy of $\lambda[X]$ forms a Banach operator ideal.

The consideration of the above relations continues the theme begun in [2] and [7]. A similar result to our main result is proven in [7] under a different assumption on the space $X$ - an isometric assumption. We do not know whether the hypothesis on $X$ in the present paper is strictly weaker than that in the previous paper. But in this case it is an isomorphic assumption and easier to verify. For example, it is satisfied by any space with a symmetric basis.

1. Some prerequisites. A. Sequence spaces. The space of all sequences of scalars $\left(s_{i}\right)$ (real or complex) with the product topology is denoted by $\omega$. The subspace of $\omega$ which contains all sequences which are eventually 0 is denoted by $\varphi$. A Banach space $\lambda$ of sequences is called a $B K$-space if the inclusion from $\lambda$ into $\omega$ is continuous. A space of sequences $\lambda$ is called normal if whenever $\left(s_{l}\right)$ is in and $\left(t_{i}\right)$ is in $m$, the $B K$-space of bounded sequences it follows that $\left(t_{t} s_{t}\right)$ is also in $\lambda$. It is known that if $\lambda$ is a $B K$ space there is an equivalent norm \|\| on $\lambda$ for which 


$$
\left\|\left(t_{t} s_{t}\right)\right\| \leqq \sup _{\imath}\left|t_{t}\right|\left\|\left(s_{i}\right)\right\|
$$

for $\left(t_{i}\right)$ in $m$.

If $\lambda$ is any set of sequences $\lambda^{\alpha}$ consists of all sequences $\left(t_{i}\right)$ such that $\left(t_{t} s_{l}\right)$ is in 1 for each $\left(s_{t}\right)$ in $\lambda$. Here 1 denotes the $B K$-space of all sequences $\left(u_{\imath}\right)$ such that $\Sigma_{l}\left|u_{l}\right|<\infty$. If $\lambda$ is a normal $B K$-space in which $\varphi$ is dense then $\lambda^{\alpha}$ is isomorphic to the topological dual space of $\lambda$ by means of the correspondence of $f$ in $\lambda^{\prime}$ to $\left(f\left(e_{n}\right)\right)$ in $\lambda^{\alpha}$. Here $e_{n}$ denotes the sequence with 1 in the $n$th place and 0 's elsewhere. The closed unit ball $U_{\lambda^{\alpha}}$ of $\lambda^{\alpha}$ for $\lambda$ a normal $B K$ space is equal to the set of all $\left(t_{i}\right)$ in $\omega$ such that $\left|\Sigma_{i} t_{t} s_{l}\right| \leqq 1$ for each $\left(s_{i}\right)$ in $\varphi$ with $\left\|\left(s_{i}\right)\right\|_{\lambda} \leqq 1$. Therefore, $U_{\lambda^{\alpha}}$ is compact in $\omega$. For $\lambda$ a normal $B K$ space and $X$ any Banach space $\lambda[X]$ denotes the space of all sequences $\left(x_{n}\right)$ in $X$ such that $\left(\left\|x_{n}\right\|_{X}\right)$ is in $\lambda$. With the norm

$$
\left\|\left(x_{n}\right)\right\|_{\lambda}=\left\|\left(\left\|x_{n}\right\|_{X}\right)\right\|_{\lambda}
$$

$\lambda[X]$ is known to be a Banach space. The closed linear span of $\varphi$ in $m$ consists of all sequences which converge to 0 and is denoted by $c_{0}$. References: [5], [6], [7].

B. Operator ideals. Let $L$ denote the class of all continuous linear mappings between Banach spaces. For two Banach spaces $E$ and $F$ let $L(E, F)$ denote the space of all continuous linear mappings between $E$ and $F$. A subclass $\mathscr{A}$ of $L$ is called an operator ideal if it is closed under sums and by multiplication on the left and right by members of $L$ where multiplication and addition is restricted to pairs of operators for which these operations are meaningful. An operator ideal $\mathscr{A}$ is called a Banach operator ideal if there is a nonnegative correspondence $\alpha$ defined on $\mathscr{A}$ such that

(1) For every pair $E, F, \mathscr{A}(E, F)=(\mathscr{A} \cap L(E, F))$ is a Banach space with norm $\alpha$.

(2) $\alpha(S T) \leqq \alpha(S)\|T\|$ if $S \in \mathscr{A}(F, G), T \in L(E, F)$.

(3) $\alpha(S T) \leqq\|S\| \alpha(T)$ if $S \in L(F, G), T \in \alpha(E, F)$.

Here \|\| denotes the uniform operator. With the uniform operator topology, $L$ is a Banach operator ideal. Let $U(E, F)$ denote the unit ball of $L(E, F)$ with the uniform norm.

The class $\mathscr{F}$ of finite rank mappings between Banach spaces is an operator ideal. Every finite rank mapping $T$ from $E$ to $F$ has a nonunique representation

$$
T x=\sum_{i=1}^{n} x_{i}^{\prime}(x) y_{i}
$$


with each $x_{i}^{\prime}$ in $E^{\prime}$ the topological dual space of $E$ and each $y_{i}$ in $F$. If $E=F$ the number

$$
\operatorname{tr}(T)=\sum_{i=1}^{n} x_{i}^{\prime}\left(y_{i}\right)
$$

does not depend on the representation of $T$; it is called the trace of $T$.

In this paper we shall refer to the following three operator ideals:

(1) The class int of all integral mappings between Banach spaces. A mapping $T$ in $L(E, F)$ is integral if there is $K>0$ such that

$$
|\operatorname{tr}(S T)| \leqq K\|S\| \quad S \in \mathscr{F}(F, E) .
$$

The norm on $L(E, F)$ is given by

$$
\|T\|=\sup \{\operatorname{tr}(S T): S \in \mathscr{F}(F, E), \quad\|S\| \leqq 1\} .
$$

(2) The class $N$ of nuclear mappings. A mapping $T$ in $L(E, F)$ is nuclear if $T=\sum_{l=1}^{\infty} T_{i}$ has rank one and $\sum_{l=1}^{\infty}\left\|T_{i}\right\|<\infty$. The norm on $N(E, F)$ is given by

$$
\|T\|_{N}=\inf \left\{\sum_{i=1}^{\infty}\left\|T_{i}\right\|: \sum_{i=1}^{\infty} T_{i}=T, \text { each } T_{\imath} \text { has rank one }\right\} .
$$

(3) The class AS of absolutely summing mappings. A mapping $T$ in $L(E, F)$ is absolutely summing if

$$
\sum_{n}\left\|T x_{n}\right\|
$$

whenever

$$
\sum_{i}\left|x^{\prime}\left(x_{n}\right)\right|<\infty \text { for all } x^{\prime} \text { in } E^{\prime}
$$

The norm on $A S(E, F)$ is given by

$$
\|T\|_{A S}=\sup \left\{\sum_{n}\left\|T x_{n}\right\|: \sum_{n}\left|x^{\prime}\left(x_{n}\right)\right| \leqq 1 \forall x^{\prime} \in U_{E^{\prime}}\right\} .
$$

Here $U_{E^{\prime}}$ denotes the unit ball in $E^{\prime}$.

References: [1], [4], [8].

2. Mappings which factor through $X$. For $X$ a Banach space let $\langle X\rangle$ denote the class of all continuous linear mappings which factor through $X$. That is, a mapping $T$ in $L(E, F)$ is in $\langle X\rangle$ if $T=T_{1} T_{2}$ 
where $T_{1}$ is in $L(X, F)$ and $T_{2}$ is in $L(E, X)$. One can show that $\langle X\rangle$ is an operator ideal if and only if $X \times X$ is isomorphic to a complemented subspace of $X$.

For $X$ a Banach space and $\left(t_{n}\right)$ a sequence of scalars the sequence $\left(t_{n} x_{n}\right)$ is in $l[X]$ for all $\left(x_{n}\right)$ in $m[X]$ if and only if $\left(t_{n}\right)$ is in $l$. We denote by $\operatorname{diag}(X)$ the collection of all such scalar diagonal mappings from $m[X]$ to $l[X]$.

2.1. Proposition. The smallest Banach operator ideal which contains $\langle X\rangle$ is equal to the class of all $T$ in $L$ which have as a factor a mapping from $\operatorname{diag}(X)$. In other words, $T$ in $L(E, F)$ is in this ideal if and only if

$$
T=T_{1} \Delta T_{2}
$$

where $T_{2}$ is in $L(E, m[X]), \Delta$ is in $\operatorname{diag}(X)$ and $T_{1}$ is in $L(l[X], F)$.

Proof. Let $\{X\}$ denote the smallest Banach operator ideal which contains $\langle X\rangle$. We first show that $T$ in $L(E, F)$ is in $\{X\}$ if and only if

$$
T=\sum_{n} S_{n} V_{n} \quad \sum_{n}\left\|S_{n}\right\|\left\|V_{n}\right\|<\infty
$$

where each $S_{n}$ is in $L(X, F)$ and each $V_{n}$ is in $L(E, X)$. It is a routine task to verify that the class $[X]$ of all such mappings does form a Banach operator ideal with the norm

$$
\|T\|=\inf \left\{\sum_{n}\left\|S_{n}\right\|\left\|V_{n}\right\|: \sum_{n} S_{n} V_{n}=T\right\},
$$

and it is clear that $[X]$ contains $\langle X\rangle$ and hence $\{X\}$.

On the other hand, for each $S$ in $L(X, F)$ the correspondence $V$ to $S V$ is a continuous linear mapping from $L(E, X)$ into $\{X\}(E, F)$ so that

$$
\sup \left\{\|S V\|_{\{X\}}: V \in U(E, X)\right\}<\infty
$$

by the Uniform Boundedness Principle. A second application of this principle shows that the set

$$
\{S V: V \in U(E, X), \quad S \in U(X, F)\}
$$

is bounded in $\{X\}$. Thus if

$$
\sum_{n}\left\|S_{n}\right\|\left\|V_{n}\right\|<\infty, \quad S_{n} \in L(X, F), \quad V_{n} \in L(E, X)
$$


it follows that

$$
\sum_{n}\left\|S_{n} V_{n}\right\|_{\{X\}}<\infty
$$

Therefore, every $T$ of the form (2.1) is in $\{X\}$.

If $T$ has the form (3.1) let $t_{n}=\left\|S_{n}\right\|\left\|V_{n}\right\|$ for each $n$. Define $T_{2}$ in $L(E, m[X])$ by

$$
T_{2} x=\left(V_{n}(x) /\left\|V_{n}\right\|\right)
$$

$\Delta$ from $m[X]$ into $l(X)$ by

$$
\Delta\left(u_{n}\right)=\left(t_{n} u_{n}\right)
$$

and $T_{1}$ from $l[X]$ into $F$ by

$$
T_{1}\left(u_{n}\right)=\sum_{n} S_{n} u_{n} /\left\|S_{n}\right\|
$$

Then $T=T_{1} \Delta T_{2}$ where $\Delta$ is in $\operatorname{diag}(X)$. On the other hand we can verify that every mapping $T$ of the form $T=T_{1} \Delta T_{2}$ has the form (2.1) by a routine inversion of the above argument.

The following theorem is proven in [3].

2.2. TheOrem. Let $\lambda$ be a normal $B K$-space containing $\varphi$. For each sequence $\left(r_{n}\right)$ in $l^{1}$ we can find sequences $\left(s_{n}\right)$ in $\lambda^{\circ}$ (the closure of $\varphi$ in $S$ ) and $\left(t_{n}\right)$ in $\lambda^{\alpha \circ}$ such that $s_{n} t_{n}=r_{n}$ for all $n$.

2.3. THEOREM. If $X$ contains a complemented subspace isomorphic to $\lambda[X]$ for $\lambda$ a normal $B K$-space containing $\varphi$ then $\langle X\rangle$ is a Banach operator ideal. $\quad(\langle X\rangle=\{X\})$

Proof. Given $T$ in $\{X\}(E, F)$ we show that $T$ factors through $X$. Since $\lambda[X]$ is complemented in $X$ it suffices to show that $T$ factors through $\lambda[X]$. By Proposition 2.1 there are $T_{1}$ in $L\left(l^{1}[X], F\right), T_{2}$ in $L(E, m[X])$ and $\left(r_{n}\right)$ in $l$ such that

$$
T x=\sum_{n} r_{n} T_{1} T_{2} x \quad x \in E .
$$

We may assume that $r_{n} \geqq 0$ for all $n$. By Theorem 2.2 there is $\left(s_{n}\right)$ in $\lambda$ and $\left(t_{n}\right)$ in $\lambda^{\alpha}$ such that $s_{n} t_{n}=r_{n}$ for all $n$. Define $R_{2}$ from $m[X]$ into $\lambda[X]$ by 


$$
R_{2}\left(u_{n}\right)=\left(s_{n} u_{n}\right)
$$

and $R_{1}$ from $\lambda[X]$ into $l[X]$ by

$$
R_{1}\left(v_{n}\right)=\left(t_{n} v_{n}\right)
$$

Then $T=T_{1} R_{1} R_{2} T_{3}$ so $T$ factors through $\lambda[X]$.

2.4. COROLlaRY. If $\lambda$ is a symmetric $B K$ space then $\langle\lambda[X]\rangle$ is an ideal for every Banach space $X$.

Proof. If $\lambda$ is a symmetric $B K$ space it is not hard to show that $\lambda[\lambda[X]]$ is isomorphic to $\lambda[X]$.

The following fact is needed later.

2.5. Proposition. If $\lambda$ is a normal $B K$-space then there is $K>0$ such that for each $\left(t_{n}\right)$ in $l$ we can find $\left(u_{n}\right)$ in $\lambda,\left(v_{n}\right)$ in $\lambda^{\alpha}$ with $\left(u_{n} v_{n}\right)=\left(t_{n}\right)$ such that

$$
\left\|\left(u_{n}\right)\right\|_{\lambda}\left\|\left(v_{n}\right)\right\|_{\lambda^{\alpha}} \leqq K \sum_{n}\left|t_{n}\right|
$$

Proof. Let $U_{1}$ denote the closed unit ball in $\lambda^{\alpha \alpha}$ and $U_{2}$ the closed unit ball in $\lambda^{\alpha}$. Then both $U_{1}$ and $U_{2}$ are compact in $\omega$ so $U_{1} U_{2}$ is compact in $\omega$ and thus closed in $l$. Since $\lambda^{\alpha \alpha} \lambda^{\alpha} \supset \lambda \lambda^{\alpha}=l$ it follows that $\bigcup_{n=1}^{\infty} n U_{1} U_{2}=l$. Using the Baire Category Theorem we can find $r>0$ such that $r U \subset U_{1} U_{2}$ where $U$ denotes the unit ball of $l$.

Given $\left(t_{n}\right)$ in $l$ and $\epsilon>0$ let $\left(r_{n}\right)$ in $c_{0}$ and $\left(s_{n}\right)$ in $l$ be such that $\left(r_{n} s_{n}\right)=\left(t_{n}\right)$ for each $n$ and $\left|r_{n}\right| \leqq 1$ for all $n$ and $\Sigma_{n}\left|s_{n}\right| \leqq \Sigma_{n}\left|t_{n}\right|+\epsilon$. Let $\left(u_{n}^{\prime}\right)$ in $\lambda^{\alpha \alpha}$ and $\left(v_{n}\right)$ in $\lambda^{\alpha}$ be such that

$$
\left(u_{n}^{\prime} v_{n}\right)=\left(s_{n}\right) \quad\left\|\left(u_{n}^{\prime}\right)\right\|\left\|\left(v_{n}\right)\right\| \leqq 1 / r .
$$

For each $\left(w_{n}\right)$ in $\varphi,\left(w_{n} u_{n}^{\prime}\right)$ is in $\varphi$. Since $c_{0}$ is the closure of $\varphi$ in $m,\left(r_{n} u_{n}^{\prime}\right)$ is in the closure of $\varphi$ in $\lambda^{\alpha \alpha}$ so $\left(r_{n} u_{n}^{\prime}\right)$ is in $\lambda$. Since $\lambda^{\alpha \alpha}$ is normal

$$
\left\|\left(r_{n} u_{n}^{\prime}\right)\right\|_{\lambda}^{\alpha \alpha} \leqq C\left\|\left(u_{n}\right)\right\|_{\lambda}{ }_{\lambda \alpha}
$$

where $C$ depends only on the norm on $\lambda$. Thus we have

$$
\left(r_{n} u_{n}^{\prime} v_{n}\right)=\left(r_{n} s_{n}\right)=\left(t_{n}\right)
$$




$$
\begin{aligned}
\left\|\left(r_{n} u_{n}^{\prime}\right)\right\|_{\lambda}\left\|\left(v_{n}\right)\right\|_{\lambda^{\alpha}} & \leqq C\left\|\left(u_{n}\right)\right\|_{\lambda^{\alpha \alpha}}\left\|\left(v_{n}\right)\right\|_{\lambda^{\alpha}} \\
& \leqq C / r \sum_{n}\left|s_{n}\right| \\
& \leqq C / r\left(\sum_{n}\left|t_{n}\right|+\epsilon\right) .
\end{aligned}
$$

Since this inequality holds for all $\epsilon>0,(2.2)$ holds with $K=C / r$.

\section{Local immersion and series immersion.}

3.1. Definition. A normed space $E$ is said to be locally immersed in a normed space $X$ if the following condition holds:

(LIX) There is a number $K>0$ such that for each finite dimensional subspace $G$ of $E$ there is a continuous linear mapping $T$ in $U(E, X)$ such that

$$
\|T x\| \geqq K\|x\| \quad x \in G
$$

3.2. Proposition. The following property ("splits through $X$ ") is equivalent to (LIX).

$(\mathrm{Sp} X)$ There is $K \geqq 1$ such that each finite rank mapping from a normed space $D$ to $E$ can be factored

$$
V=V_{1} V_{2} V_{3} ;\left\|V_{1}\right\|\left\|V_{2}\right\|\left\|V_{3}\right\| \leqq K\|V\|
$$

with $V_{3}$ in $L(D, E), V_{2}$ in $L(E, Y)$ where $Y$ is a closed subspace of $X$ and $V_{1}$, in $L(Y, E)$.

Proof. $\quad(\operatorname{Sp} X) \Rightarrow(\mathrm{LIX})$. Let $V$ denote the inclusion map from $G$ into $E$, and let $V_{1}, V_{2}, V_{3}$ satisfy $(\operatorname{Sp} X)$. If $T=V_{2} V_{3} /\left\|V_{2} V_{3}\right\|$ then $\|T\|=1$, and for each $x$ in $G$ we have

$$
\begin{aligned}
\|x\| & =\left\|V_{1} V_{2} V_{3} x\right\| \leqq\left\|V_{1}\right\|\left\|V_{2} V_{3} x\right\| \\
& \leqq\left\|V_{1}\right\|\left\|V_{2}\right\|\left\|V_{3}\right\|\|T x\| \leqq K\|T x\| .
\end{aligned}
$$

$(\mathrm{LIX}) \Rightarrow(\operatorname{Sp} X)$. Let $G=V(D), V_{3}=V$ and $V_{2}=T$ where $T$ is given by (LIX). Let $Y=T(G)$ and define $V_{3}$ on $Y$ by $V_{3} y=x$ if $T x=y$. Then

$$
V=V_{1} V_{2} V_{3} \text { and }\left\|V_{1}\right\|\left\|V_{2}\right\|\left\|V_{3}\right\| \leqq K\|V\| \text {. }
$$


3.3. Definition. A normed space $E$ is said to be series immersed in a normed space $X$ if the following statement holds:

(SIX) For each absolutely divergent series $\Sigma_{n} x_{n}$ in $E$ there is $T$ in $L(E, F)$ such that $\Sigma_{n} T x_{n}$ diverges absolutely.

We omit the proof of the following statement which is known [8].

3.4. LeMmA. For $T$ a continuous linear mapping from $c_{0}$ into a normed space $E$ the following statements are equivalent:

(a) $T$ is nuclear;

(b) $T$ is integral;

(c) $T$ is absolutely summing;

(d) $\Sigma_{n}\left\|T e_{n}\right\|<\infty$.

3.5. Proposition. For $E$ and $X$ arbitrary Banach spaces the following conditions are equivalent to (SIX) and thus [7] implied by (LIX).

(ASX) For every Banach space $D$ a mapping $T$ in $L(D, E)$ is absolutely summing if ST is absolutely summing for all $S$ in $L(E, X)$.

$(\mathrm{ASX})_{0}$ The same statement as (a) with $D=c_{0}$.

Proof. (SIX) $\Rightarrow($ ASX). Suppose $T$ in $L(D, E)$ is not absolutely summing. Then there is a weakly absolutely summable series $\sum_{n} x_{n}$ in $D$ such that $\Sigma_{n}\left\|T x_{n}\right\|=\infty$. By (SIX) there is $S$ in $L(E, X)$ such that $\Sigma_{n}\left\|S T x_{n}\right\|=\infty$ so that ST is not absolutely summing.

$(\mathrm{ASX}) \Rightarrow(\mathrm{ASX})_{0} . \quad$ Clear.

$(\mathrm{ASX})_{0} \Rightarrow(\mathrm{SIX}) . \quad$ Suppose $\Sigma_{n} x_{n}$ is a series in $E$ with $\Sigma_{n}\left\|x_{n}\right\|=$ $\infty$. If $\sum_{n} x_{n}$ is not weakly absolutely summable it is easy to find $T$ in $L(E, X)$ such that $\Sigma_{n}\left\|T x_{n}\right\|=\infty$ where $T$ has rank one. If $\sum_{n} x_{n}$ is weakly absolutely summable define $T$ from $c_{0}$ into $E$ by

$$
T\left(\left(t_{n}\right)\right)=\Sigma_{n} t_{n} x_{n}
$$

By 3.4, $T$ is not absolutely summing because $\Sigma_{n}\left\|T e_{n}\right\|=\Sigma_{n}\left\|x_{n}\right\|=\infty$ so by $(\mathrm{ASX})_{0}$ there is $S$ in $L(E, X)$ such that $\mathrm{ST}$ is not absolutely summing. Consequently by 3.4 ,

$$
\sum_{n}\left\|S T e_{n}\right\|=\sum_{n}\left\|S x_{n}\right\|=\infty
$$

3.6. Proposition. For Banach spaces $E$ and $X$ the following condition is implied by (LIX) and implies (SIX): 
(int X) A mapping $T$ from a Banach space $D$ into $E$ is integral if $\mathrm{ST}$ is integral for all $S$ in $L(E, Y)$ as $Y$ ranges over the closed subspaces of $X$.

Proof. (LIX) $\Rightarrow$ (int X). Suppose ST is integral for all $S$ in $L(E, Y)$. We first show there is $M>0$ such that

(3.1) $\sup \{\|S T\|: S \in U(E, Y), Y$ is a closed subspace of $X\} \leqq M$.

Let $Y$ denote the set of all closed subspaces $Y$ of $X$. Let $Z_{1}(\mathscr{Y})$ denote the Banach space of all indexed families $\left(S_{Y}\right)_{Y \in Q}$ where $S_{Y}$ is in $L(E, Y)$ and $\sup _{Y}\left\|S_{Y}\right\|=\left\|\left(S_{Y}\right)\right\|<\infty$. Let $Z_{2}(\mathscr{Y})$ denote the Banach space of all indexed families $\left(V_{Y}\right)_{Y \in \mathscr{y}}$ where $V_{Y}$ is in $\operatorname{int}(D, Y)$ and $\sup _{Y}\left\|V_{Y}\right\| \|=$ $\left\|\left(V_{Y}\right)\right\|<\infty$. The correspondence $\left(S_{Y}\right)_{Y \in \mathscr{Y}} \rightarrow\left(S_{Y} T\right)_{Y \in \mathscr{y}}$ determines a linear mapping from $Z_{1}(\mathscr{Y})$ into $Z_{2}(\mathscr{Y})$ which is continuous by the Closed Graph Theorem. There is thus $M>0$ such that

$$
\left\|\left(S_{Y} T\right)\right\| \leqq M\left\|\left(S_{Y}\right)\right\| \quad\left(S_{Y}\right) \in Z_{1}(\mathscr{Y})
$$

which proves (3.1).

If $S$ is a finite rank mapping in $L(E, D)$ let $V$ denote the inclusion from $\operatorname{TS}(E)$ into $E$. By $(\operatorname{Sp} X), V=V_{1} V_{2} V_{3}$ with $V_{3}$ in $L(T S(E), E), T_{2}$ in $L(E, Y)$ where $Y$ is a subspace of $X, V_{1}$ is in $L(Y, E)$ and $\left\|V_{1}\right\|\left\|V_{2}\right\|\left\|V_{3}\right\| \leqq K$. Thus we have

$$
\begin{aligned}
|\operatorname{tr} T S| & =\left|\operatorname{tr}\left(V_{1} V_{2} V_{3} T S\right)\right| \\
& \leqq\left\|V_{1}\right\|\|\| V_{2} V_{3} T\|\| S \| \\
& \leqq\left\|V_{1}\right\| M\left\|V_{2}\right\|\left\|V_{3}\right\|\|S\| \leqq M K\|S\|
\end{aligned}
$$

which shows $T$ is integral.

$$
\text { (int } X) \Rightarrow(\text { SIX) by Lemma } 3.4 \text { and Proposition 3.5. }
$$

Notice the connection of the following statement with the results of $\S 2$.

3.7. Proposition. The normed space $E$ is series immersed in the Banach space $X$ if and only if the following condition holds:

( $\operatorname{diag} X)$ There is $M>0$ such that for each finite dimensional subspace $F$ of $E$ one can find a mapping $R$ from $E$ into $m[X]$ and $a$ mapping $\Delta$ in $\operatorname{diag}(X)$ such that

$$
\begin{gathered}
\|\Delta R x\| \leqq\|x\| \text { for } x \text { in } E \\
M\|\Delta R x\| \geqq\|x\| \text { for } x \text { in } F .
\end{gathered}
$$


Proof. $\quad(\operatorname{diag} X) \Rightarrow(\mathrm{SIX}) . \quad$ If $\Sigma_{n}\left\|T x_{n}\right\|<\infty$ for all $T$ in $L(E, X)$ then there is $K>0$ such that $\Sigma_{n}\left\|T x_{n}\right\| \leqq K\|T\|$ for each $T$ in $L(E, X)$ by the Uniform Boundedness Principle. For $k$ a fixed positive integer let $R$ and $\Delta$ satisfy $(\operatorname{diag} X)$ for the finite dimensional subspace spanned by $\left\{x_{1}, x_{2}, \cdots, x_{k}\right\}$. There is a bounded sequence $\left(T_{1}\right)$ in $L(E, X)$, such that $R x=\left(T_{1} x\right)$ and a sequence $t_{l}(\geqq 0)$ in $l$ such that $\Delta\left(y_{l}\right)=\left(t_{1} y_{1}\right)$. Since $\|\Delta R x\| \leqq\|x\|$ for $x$ in $E$ it follows that $\Sigma_{l} t_{i}\left\|T_{i}\right\| \leqq 1$. Thus we have

$$
\begin{aligned}
\sum_{n=1}^{k}\left\|x_{n}\right\| & \leqq M \sum_{n=1}^{k}\left\|\Delta R x_{n}\right\|=M \sum_{n=1}^{k} \sum_{i} t_{i}\left\|T_{i} x_{n}\right\| \\
& \leqq M \sum_{i} \sum_{n=1}^{k} t_{i}\left\|T_{i} x_{n}\right\| \leqq M K \sum_{i} t_{i}\left\|T_{i}\right\| \\
& \leqq M K .
\end{aligned}
$$

Since $M$ and $K$ are independent of $k, \Sigma_{n}\left\|x_{n}\right\|<\infty$.

$(\mathrm{SIX}) \Rightarrow($ int X). We proceed as in [2]. Let $\sigma(E)$ consist of all sequences $\left(x_{n}\right)$ in $E$ such that $\Sigma_{n}\left\|T x_{n}\right\|<\infty$ for all $T$ in $L(E, X)$. Then $\sigma(E)$ is a Banach space with the norm

$$
\left\|\left(x_{n}\right)\right\|_{X}=\sup \left\{\sum_{n}\left\|T x_{n}\right\|:\|T\| \leqq 1\right\} .
$$

If (SIX) holds $\sigma(E)=l[E]$ so there is $M^{\prime}>0$ such that

$$
\sum_{n}\left\|x_{n}\right\| \leqq(1 / 2) M^{\prime}\left\|\left(x_{n}\right)\right\|_{X}
$$

for all $\left(x_{n}\right)$ in $l[E]$. From this one concludes that for $\left(x_{n}\right)$ in $l[E]$

$$
\sum_{n}\left\|x_{n}\right\| \leqq(M / 2) \sup \left\{\sum_{n} \varphi_{n}\left(T x_{n}\right): \varphi_{n} \in U_{X}^{\circ}, T \in U(E, X)\right\}
$$

The topological dual space of $l[E]$ can be represented by $m\left[E^{\prime}\right]$ with duality given by the bilinear form

$$
\left\langle\left(x_{\jmath}^{\prime}\right),\left(x_{\jmath}\right)\right\rangle=\sum_{j} x_{j}^{\prime}\left(x_{j}\right) \quad\left(x_{\jmath}^{\prime}\right) \in m\left[E^{\prime}\right] ; \quad\left(x_{j}\right) \in l[E] .
$$

From (3.2) it follows that the unit ball of $m\left[E^{\prime}\right]$ is contained in the $w^{*}$-closed convex cover of sequences having the form $\left(M^{\prime} / 2\right)\left(T^{\prime} \varphi_{l}\right)$ where $\left\|T^{\prime}\right\| \leqq 1$ and $\left\|\varphi_{1}\right\| \leqq 1$ for each $j$.

For any finite subset $A=\left\{x_{1}, x_{2}, \cdots, x_{k}\right\}$ of $E$ not containing 0 , let $\eta=\left(x_{1}^{\prime}, x_{2}^{\prime}, \cdots, x_{k}^{\prime}, 0,0, \cdots\right)$ be such that $x_{n}^{\prime}\left(x_{n}\right)=\left\|x_{n}\right\|$ and $x_{n}^{\prime}=1$ for $n=1,2, \cdots, k$. By the preceding paragraph we can find $T_{1}, \cdots, T_{r}$ in $U(E, X), c_{1}, \cdots, c_{r} \geqq 0$ with $\sum_{i=1}^{r} c_{1}=1$ and $\left(\varphi_{i j}\right)_{j=1}^{\infty} i=1,2, \cdots, r$ with each $\varphi_{i j}$ in the unit ball of $X^{\prime}$ such that 


$$
\begin{aligned}
& \left|\left\langle\eta-\sum_{i=1}^{r} c_{l}\left(M^{\prime} / 2\right)\left(T_{i}^{\prime} \varphi_{i j}\right), x_{n} e_{n}\right\rangle\right| \\
& <(1 / 2) \min \left\{\left\|x_{n}\right\|: n=1,2, \cdots, k\right\}
\end{aligned}
$$

for each $n=1,2, \cdots, k$. Here $x_{n} e_{n}$ is the sequence with $x_{n}$ in the $n$th place and 0's elsewhere. From (4.2) we see that for each $n$

$$
\left|\left\|x_{n}\right\|-(M / 2) \sum_{i=1}^{r} c_{\imath} T_{i}^{\prime} \varphi_{i n}\left(x_{n}\right)\right|<(1 / 2)\left\|x_{n}\right\|
$$

from which it follows that

$$
\begin{aligned}
M^{\prime} \sum_{i=1}^{r} c_{l}\left\|T_{i} x_{n}\right\| & \geqq M^{\prime} \sum_{i=1}^{r} c_{l}\left|T_{i}^{\prime} \varphi_{i n}\left(x_{n}\right)\right| \\
& >\left\|x_{n}\right\| \text { for } n=1,2, \cdots, k .
\end{aligned}
$$

If $F$ is a finite dimensional subspace of $E$ let $A$ be a $\left(2 M^{\prime}\right)^{-1}$-net for the unit sphere of $F$. If $T_{1}, T_{2}, \cdots, T_{r}$ and $c_{1}, c_{2}, \cdots, c_{r}$ satisfy (3.4) define $R$ from $E$ into $m[X]$ by

$$
R x=\left(T_{1} x, T_{2} x, \cdots, T_{r} x, 0,0, \cdots\right)
$$

and $\Delta$ from $m[X]$ into $l[X]$ by

$$
\Delta\left(y_{j}\right)=\left(c_{1} y_{1}, \cdots, c_{r} y_{r}, 0,0, \cdots\right) \text {. }
$$

Then $\|\Delta R x\| \leqq\|x\|$ for $x$ in $E$ since $\left\|T_{i}\right\| \leqq 1$ for each $i$ and $\Sigma_{i}\left|c_{i}\right|=$ 1. For $x$ in $F$ with $\|x\|=1$ there is $y$ in $A$ with $\|x-y\|<\left(2 M^{\prime}\right)^{-1}$ so that

$$
\begin{aligned}
\|\Delta R x\| & \geqq(\|\Delta R y\|-\|\Delta R(y-x)\| \\
& \geqq\left(M^{\prime}\right)^{-1}\|y\|-\|y-x\| \geqq\left(2 M^{\prime}\right)^{-1} .
\end{aligned}
$$

Therefore, the second inequality of $(\operatorname{diag} X)$ holds with $M=\left(2 M^{\prime}\right)^{-1}$.

3.8. THEOREM. Let $X$ be a Banach space which contains a subspace isomorphic to $\lambda[X]$ where $\lambda$ is a normal BK-space. For $E$ any Banach space the following statements are equivalent:

(SIX), (LIX), (ASX), (int X).

Proof. $\quad(\mathrm{SIX}) \Rightarrow(\mathrm{LIX})$. It suffices to prove that $E$ is locally immersed in $\lambda[X]$. Given $F$ a finite dimensional subspace of $E$ let $R$ and $\Delta$ be determined by $(\operatorname{diag} X)$ of Proposition 3.7.

If $\Delta\left(y_{n}\right)=\left(t_{n} y_{n}\right)$ for $\left(y_{n}\right)$ in $m[X]$ let $t_{n}=u_{n} v_{n}$ where $\left(u_{n}\right)$ is in $\lambda,\left(v_{n}\right)$ is in $\lambda^{\alpha}$ and 


$$
\left\|\left(u_{n}\right)\right\|_{\lambda} \leqq 1, \quad\left\|\left(v_{n}\right)\right\|_{\lambda^{\alpha}} \leqq K
$$

where $K$ depends only on $\lambda$ (Proposition 2.5). If $R x=\left(R_{n} x\right)$ for $x$ in $E$ define $T$ from $E$ into $\lambda[X]$ by

$$
T x=\left(u_{n} R_{n} x\right) .
$$

Since $\left\|\left(u_{n}\right)\right\|_{\lambda} \leqq 1$

$$
\|T x\|_{\lambda[X]}=\left\|\left(\left\|u_{n} R_{n} x\right\|\right)\right\|_{\lambda} \leqq \sup _{n}\left\|R_{n} x\right\|\left\|\left(u_{n}\right)\right\|_{\lambda} \leqq 1 .
$$

For $x$ in $F$

$$
\|T x\|_{\lambda[X]}=\left\|\left(\left\|u_{n} R_{n} x\right\|\right)\right\|_{\lambda} \geqq K^{-1} \sum v_{n}\left\|u_{n} R_{n} x\right\|
$$

since the function defined by $f\left(\left(s_{n}\right)\right)=\Sigma_{n} v_{n} s_{n}$ is in $\lambda^{\prime}$ and $\|f\| \leqq K$. Thus

$$
\begin{aligned}
\|T x\|_{\lambda[X]} & \geqq K^{-1} \sum v_{n}\left\|u_{n} R_{n} x\right\| \\
& =K^{-1}\left\|\left(t_{n}\left\|R_{n} x\right\|\right)\right\|_{l} \\
& \geqq M K^{-1}\|x\| .
\end{aligned}
$$

Therefore, $E$ is locally immersed in $X$.

(LIX) $\Leftrightarrow($ ASX) by Proposition 3.5.

$(\mathrm{LIX}) \Rightarrow($ int $X) \Rightarrow$ (SIX) by Proposition 3.6.

\section{REFERENCES}

1. Y. Gordon, D. R. Lewis and J. R. Retherford, Banach ideals of operators with applications, J. Functional Analysis, 14 (1973), 85-129.

2. N. J. Kalton and W. H. Ruckle, A series characterization of subspaces of $L_{p}(\mu)$, BAMS 79 (1973), 1019-1022.

3. R. E. Jamison and W. H. Ruckle, Factoring absolutely convergent series, to appear.

4. A. Pietsch, Ideale von $S_{p}$-Operatoren in Banachräumen, Studia Math., 38 (1970), 59-69.

5. W. H. Ruckle, On the construction of sequence spaces that have Schauder Bases, Canad. J. Math., 18 (1966), 1281-1293.

6. - Representation and series summability of complete biorthogonal sequences, Pacific J. Math., 34 (1970), 511-528.

7. - Absolutely divergent series and isomorphism of subspaces, Pacific J. Math., 56 (1975), in print.

8. J. R. Retherford and C. Stegall, Fully nuclear and completely nuclear operators with application to $\mathscr{L}_{1}$ and $\mathscr{L}_{\infty}$ spaces, TAMS, 163 (1972), 457-492.

Received November 14, 1975. 



\section{Pacific Journal of Mathematics}

Vol. 68, No. 1

March, 1977

Richard Julian Bagby, On $L^{p}, L^{q}$ multipliers of Fourier transforms . .......

Robert Beauwens and Jean-Jacques Van Binnebeek, Convergence theorems in

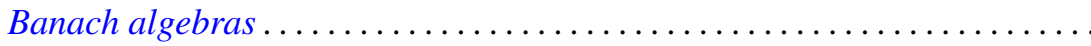

James Cyril Becker, Skew linear vector fields on spheres in the stable

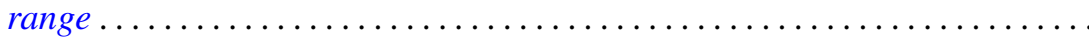

Michael James Beeson, Continuity and comprehension in intuitionistic formal

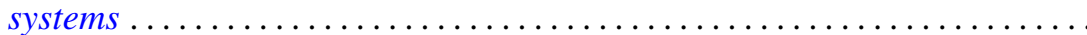

James K. Deveney, Generalized primitive elements for transcendental field

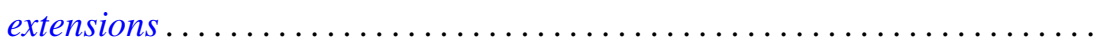

Samuel S. Feder, Samuel Carlos Gitler and K. Y. Lam, Composition properties

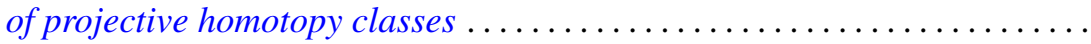

Nathan Jacob Fine, Tensor products of function rings under composition ......

Benno Fuchssteiner, Iterations and fixpoints . . . . . . . . . . . . . .

Wolfgang H. Heil, On punctured balls in manifolds

Shigeru Itoh, A random fixed point theorem for a multivalued contraction

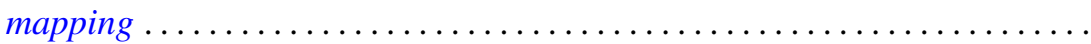

Nicolas P. Jewell, Continuity of module and higher derivations . . . . . . ......

Roger Dale Konyndyk, Residually central wreath products . . . . . . . . . . .

Linda M. Lesniak and John A. Roberts, On Ramsey theory and graphical

parameters.

Vo Thanh Liem, Some cellular subsets of the spheres.

Dieter Lutz, A perturbation theorem for spectral operators

P. H. Maserick, Moments of measures on convex bodies ... . . .

Stephen Joseph McAdam, Unmixed 2-dimensional local domains . .

D. B. McAlister and Norman R. Reilly, E-unitary covers for inverse semigroups...

William H. Meeks, III and Julie Patrusky, Representing codimension-one

homology classes by embedded submanifolds . . .

Premalata Mohapatro, Generalised quasi-Nörlund summability . .

Takahiko Nakazi, Superalgebras of weak-*Dirichlet algebras .

Catherine Louise Olsen, Norms of compact perturbations of operators .

William Henry Ruckle, Absolutely divergent series and isomorphism of

subspaces. II.

Bernard Russo, On the Hausdorff-Young theorem for integral operators .

Arthur Argyle Sagle and J. R. Schumi, Anti-commutative algebras and

homogeneous spaces with multiplications ............

Robert Evert Stong, Stiefel-Whitney classes of manifolds .

D. Suryanarayana, On a theorem of Apostol concerning Möbius functions of

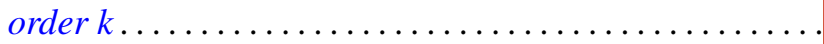

Yoshio Tanaka, On closedness of $C$ - and $C^{*}$-embeddings . . 